\section{Persistent Perianal Dermatitis Associated with Mucosal Hemorrhoidal Prolapse}

To the Editor:

Perianal dermatitis is a common acute to chronic and usually extremely pruritic eczema of the area around the anal orifice. ${ }^{1}$ It affects a humid, dark, and mechanically traumatized region that usually has a high concentration of bacteria. Any increase in moisture, such as that caused by occult and overt soiling, leads to maceration and reduced barrier function. ${ }^{2}$ Skin irritation caused by proteolytic enzymes found in rectal mucoid secretions creates erythema, edema, and pruritus, sometimes accompanied by stinging and burning sensations. Thus, any variations in the anal anatomy that produce soiling can be responsible for perianal dermatitis. ${ }^{3}$

Grade III and grade IV hemorrhoids and anal tags, polyps, fissures, and fistulas are causes of overt soiling and are evident upon dermatologic examination. ${ }^{4}$ Contrarily, internal mucosal-hemorrhoidal prolapse, another cause of occult soiling, stays in the anal canal and cannot be seen through external inspection; it can be diagnosed only with a complete proctologic examination, which includes proctoscopy. ${ }^{5}$

In our experience, the diagnosis of this mucocutaneous disorder is often delayed for months, and patients are unsuccessfully treated with various topical treatment regiments aimed at curing one (or sometimes several) of the different diagnosed conditions listed in Table 1, without elimination of the real trigger.

With the collaboration of dermatologists and colorectal surgeons during 2008, 15 patients ( 9 males and 6 females, with an average age of 51 years) were examined for

From the Department of Anesthesiology, Intensive Care and Dermatology Sciences, University of Milan, I.R.C.C.S. FoundationCa' Granda, Ospedale Maggiore Policlinico, Milan, Italy; Università degli Studi di Milano-Bicocca, Milan, Italy; Fondazione Don Carlo Gnocchi, ONLUS, IRCCS "S. Maria Nascente," Milan, Italy; and Istituti Clinici Zucchi, Monza, Italy.

Address reprint requests to Dr. Elena Guanziroli, Department of Anesthesiology, Intensive Care and Dermatology Sciences, University of Milan, I.R.C.C.S. FoundationCa' Granda, Ospedale Maggiore Policlinico, Milan, Italy. E-mail: elena.guanziroli@hotmail.it

DOI 10.2310/6620.2011.11017

(C) 2011 American Contact Dermatitis Society. All Rights Reserved. DECKER perianal dermatitis and occult anorectal diseases. They underwent complete skin inspection, proctologic examination with proctoscopy, and perianal swabs. Anorectal manometry and colonoscopy were performed to exclude fecal incontinence, bowel inflammatory diseases, and neoplasms.

The typical presentation included perianal skin maceration and various degrees of bright pink moist erythema, often accompanied by signs of inflammation, such as superficial edema, infiltration, and tenderness. The clinical signs were sometimes obscured by topical pretreatment (zinc paste, antimycotic and antibiotic ointments), but pruritus was always present. In all cases, proctologic examination revealed mucosal-hemorrhoidal prolapse.

Results of microbiologic analysis of perianal swabs were positive for seven patients. Staphylococcus aureus, which was isolated in six cultures, was the most common infectious agent.

When necessary, prolapsectomy was followed by medical treatments, which were successful in all cases. Potassium permanganate reduced erythema and pruritus in all patients, and antibiotic treatment based on swab results was prescribed for seven patients to eliminate bacterial infection. At follow-up, which ranged from 18 months to 2 years, no patients had symptom recurrences.

Clinical features, physical examination results, proctoscopy results, microbiologic analysis results, and specific treatments are summarized in Table 2.

We wish to emphasize the importance of collaboration between dermatologists and colorectal surgeons to discover and eliminate the provoking factor of perianal dermatitis. Among the different diagnostic procedures (which include patch testing, stool examination for ova and parasites, and bacteriologic and fungal cultures of stool and the perianal region), proctologic examination plays a primary role. In fact, up to $52 \%$ of patients with this cutaneous alteration have anorectal diseases, which in most cases show no other signs or symptoms. ${ }^{4}$ For this reason, every patient with an unclear perianal dermatitis needs a meticulous proctologic examination.

\section{Acknowledgments}

Financial disclosures of authors and reviewer(s): None reported. 
Table 1. Differential Diagnoses of Perianal Dermatitis

\begin{tabular}{lc}
\hline Category & Causes \\
\hline $\begin{array}{l}\text { Contact dermatitis (allergic, toxic) } \\
\text { Irritant dermatitis }\end{array}$ & $\begin{array}{c}\text { Toilet paper, cleansers, suppositories, hemorrhoidal ointments } \\
\text { Poor hygiene, soiling ( hemorrhoid, fistula, fissure), inflammatory } \\
\text { bowel diseases } \\
\text { Infectious dermatitis }\end{array}$ \\
$\begin{array}{l}\text { Candidiasis, tinea inguinalis, oxyuriasis, perianal streptococcal } \\
\text { dermatitis, condyloma acuminatum, condyloma latum, rectal } \\
\text { Neoplasm }\end{array}$ \\
gonorrhea \\
Bewen disease, extramammary Paget disease, anal basal cell \\
carcinoma \\
Lichen planus, lichen sclerosus, psoriasis, seborrheic dermatitis, \\
atopic dermatitis, Darier disease
\end{tabular}

Table 2. Demographics, Clinical Features, and Treatment of Patients with Perianal Dermatitis

\begin{tabular}{|c|c|c|c|c|c|c|c|c|}
\hline $\begin{array}{l}\text { Patient } \\
\text { No. }\end{array}$ & Sex & Age (yr) & $\begin{array}{l}\text { Physical } \\
\text { Examination } \\
\text { Results }\end{array}$ & $\begin{array}{l}\text { Proctologic } \\
\text { Examination } \\
\text { Results }\end{array}$ & Symptoms & Culture Results & Treatment & $\begin{array}{l}\text { Therapeutic } \\
\text { Response }\end{array}$ \\
\hline 1 & $\mathrm{M}$ & 19 & $\begin{array}{l}\text { Perianal erythema, } \\
\text { wet anus, } \\
\text { maceration }\end{array}$ & M-H P II & $\begin{array}{l}\text { Pruritus, } \\
\text { bleeding, } \\
\text { pain on } \\
\text { defecation }\end{array}$ & $\mathrm{N}$ & $\mathrm{P}, \mathrm{PP}$ & Good \\
\hline 2 & $\mathrm{M}$ & 61 & $\begin{array}{l}\text { Perianal erythema, } \\
\text { wet anus, } \\
\text { excoriations }\end{array}$ & M-H P II & $\begin{array}{l}\text { Pruritus, } \\
\text { burning } \\
\text { sensation }\end{array}$ & MSSA & $\begin{array}{l}\text { P, PP, amoxicillin } \\
\quad+\text { clavulanic acid }\end{array}$ & Good \\
\hline 3 & M & 43 & $\begin{array}{l}\text { Perianal erythema, } \\
\text { wet anus, } \\
\text { excoriations }\end{array}$ & M-H P II & $\begin{array}{l}\text { Pruritus, } \\
\text { burning } \\
\text { sensation }\end{array}$ & MSSA & $\begin{array}{l}\text { P, PP, amoxicillin } \\
\quad+\text { clavulanic acid }\end{array}$ & Good \\
\hline 4 & $\mathrm{M}$ & 68 & $\begin{array}{l}\text { Perianal erythema, } \\
\text { wet anus, } \\
\text { dyschromia }\end{array}$ & M-H P II & $\begin{array}{l}\text { Pruritus, } \\
\text { bleeding }\end{array}$ & $\mathrm{N}$ & $\mathrm{P}, \mathrm{PP}$ & Good \\
\hline 5 & M & 61 & $\begin{array}{l}\text { Perianal erythema, } \\
\text { wet anus }\end{array}$ & M-H P II & Pruritus & $\mathrm{N}$ & $\mathrm{P}, \mathrm{PP}$ & Good \\
\hline 6 & $\mathrm{~F}$ & 79 & $\begin{array}{l}\text { Perianal erythema, } \\
\text { wet anus, } \\
\text { maceration, } \\
\text { excoriations }\end{array}$ & M-H P II & $\begin{array}{l}\text { Pruritus, } \\
\text { burning } \\
\text { sensation, } \\
\text { bleeding }\end{array}$ & MSSA, E. coli & $\begin{array}{l}\text { P, PP, amoxicillin } \\
\quad+\text { clavulanic acid }\end{array}$ & Good \\
\hline 7 & $\mathrm{M}$ & 29 & $\begin{array}{l}\text { Perianal erythema, } \\
\text { wet anus }\end{array}$ & M-H P II & Pruritus & $\mathrm{N}$ & $\mathrm{P}, \mathrm{PP}$ & Good \\
\hline 8 & M & 69 & $\begin{array}{l}\text { Perianal erythema, } \\
\text { wet anus, } \\
\text { maceration }\end{array}$ & M-H P II & $\begin{array}{l}\text { Pruritus, } \\
\text { burning } \\
\text { sensation }\end{array}$ & $\begin{array}{l}\text { E. coli, } \\
\text { Acinetobacter } \\
\text { baumannii }\end{array}$ & $\mathrm{P}, \mathrm{PP}$, imipenem & Good \\
\hline 9 & M & 58 & $\begin{array}{l}\text { Perianal erythema, } \\
\text { wet anus, } \\
\text { excoriations }\end{array}$ & M-H P II & Pruritus & MSSA & $\begin{array}{l}\text { P, PP, amoxicillin } \\
\quad+\text { clavulanic acid }\end{array}$ & Good \\
\hline 10 & $\mathrm{~F}$ & 51 & $\begin{array}{l}\text { Perianal erythema, } \\
\text { wet anus }\end{array}$ & M-H P II & Pruritus & $\mathrm{N}$ & $\mathrm{P}, \mathrm{PP}$ & Good \\
\hline 11 & $\mathrm{~F}$ & 43 & $\begin{array}{l}\text { Perianal erythema, } \\
\text { wet anus }\end{array}$ & M-H P II & $\begin{array}{l}\text { Pruritus, } \\
\text { bleeding }\end{array}$ & $\mathrm{N}$ & $\mathrm{P}, \mathrm{PP}$ & Good \\
\hline 12 & $\mathrm{~F}$ & 27 & $\begin{array}{l}\text { Perianal erythema, } \\
\text { wet anus }\end{array}$ & M-H P II & $\begin{array}{l}\text { Pruritus, } \\
\text { bleeding }\end{array}$ & $\mathrm{N}$ & $\mathrm{P}, \mathrm{PP}$ & Good \\
\hline
\end{tabular}


Table 2. Cont.

\begin{tabular}{|c|c|c|c|c|c|c|c|c|}
\hline $\begin{array}{l}\text { Patient } \\
\text { No. }\end{array}$ & Sex & Age (yr) & $\begin{array}{l}\text { Physical } \\
\text { Examination } \\
\text { Results }\end{array}$ & $\begin{array}{l}\text { Proctologic } \\
\text { Examination } \\
\text { Results }\end{array}$ & Symptoms & Culture Results & Treatment & $\begin{array}{l}\text { Therapeutic } \\
\text { Response }\end{array}$ \\
\hline 13 & $\mathrm{~F}$ & 60 & $\begin{array}{l}\text { Perianal erythema, } \\
\text { wet anus, } \\
\text { excoriations }\end{array}$ & M-H P II & Pruritus & MRSA & $\mathrm{P}, \mathrm{PP}$, vancomycin & Good \\
\hline 14 & $\mathrm{~F}$ & 38 & $\begin{array}{l}\text { Perianal erythema, } \\
\text { wet anus, } \\
\text { excoriations }\end{array}$ & M-H P II & Pruritus & $\mathrm{N}$ & $\mathrm{P}, \mathrm{PP}$ & Good \\
\hline 15 & M & 67 & $\begin{array}{l}\text { Perianal erythema, } \\
\text { wet anus, } \\
\text { excoriations }\end{array}$ & M-H P II & $\begin{array}{l}\text { Pruritus, } \\
\text { burning } \\
\text { sensation }\end{array}$ & MSSA, E. coli & $\begin{array}{l}\mathrm{P}, \mathrm{PP}, \text { amoxicillin }+ \\
\text { clavulanic acid }\end{array}$ & Good \\
\hline
\end{tabular}

E. coli = Escherichia coli $\mathrm{F}=$ female; $\mathrm{M}=$ male; $\mathrm{M}-\mathrm{H}$ P II = mucosal-hemorrhoidal prolapse, grade II; MRSA = methicillin-resistant Staphylococcus aureus; MSSA = methicillin-sensitive Staphylococcus aureus; $\mathrm{N}=$ negative; $\mathrm{P}=$ prolapsectomy; $\mathrm{PP}=$ potassium permanganate.

\section{Elena Guanziroli}

Stefano Veraldi

Department of Anesthesiology, Intensive Care and

Dermatology Sciences, University of Milan, I.R.C.C.S.

FoundationCa' Granda, Ospedale Maggiore Policlinico, Milan, Italy

\section{Angelo Guttadauro}

Università degli Studi di Milano-Bicocca, Milan, Italy

Istituti Clinici Zucchi, Monza, Italy

\section{Gaetano Rizzitelli}

Fondazione Don Carlo Gnocchi, ONLUS, IRCCS "S. Maria Nascente," Milan, Italy, Istituti Clinici Zucchi, Monza, Italy

Silvia Frassani

Istituti Clinici Zucchi, Monza, Italy

\section{References}

1. Herbst R. Perineal streptococcal dermatitis/disease: recognition and management. Am J Clin Dermatol 2003;4:555-60, doi:10.2165/ 00128071-200304080-00005.

2. Andersen PH, Bucher AP, Saeed I, et al. Faecal enzymes: in vivo human skin irritation. Contact Dermatitis 1994;30:152-8, doi:10.1111/j.1600-0536.1994.tb00696.x.

3. Pirone E, Infantino A, Masin A, et al. Can proctological procedures resolve perianal pruritus and mycosis? A prospective study of 23 cases. Int J Colorectal Dis 1992;7:18-20, doi:10.1007/ BF01647655.

4. Siddiqi S, Vijay V, Ward M, et al. Pruritus ani. Ann R Coll Surg Engl 2008;90:457-63, doi:10.1308/003588408X317940.

5. Gabrielli F, Chiarelli M, Cioffi U, et al. Day surgery for mucosalhaemorrhoidal prolapse using a circular stapler and modified regional anaesthesia. Dis Colon Rectum 2001;44:842-4, doi:10.1007/ $\underline{\mathrm{BF} 02234705 .}$ 